Journal for Educational Research Online

Journal für Bildungsforschung Online

https://doi.org/10.31244/jero.2021.02.02

Volume 13 (2021), No. 2, 5-29

CC BY-NC-SA 4.o Waxmann 2021

Armin Jentsch, Jörg Doll, Ilse Stangen, Dennis Meyer

\& Gabriele Kaiser

\title{
Die Bedeutung von sprachbezogenen Merkmalen und von in universitären Lerngelegenheiten genutzten Studieninhalten für den Wissenserwerb von Lehramtsstudierenden
}

\section{Zusammenfassung}

In der vorliegenden Studie wird untersucht, wie sprachbezogene Merkmale von Lehramtsstudierenden und in universitären Lerngelegenheiten von ihnen genutzte Studieninhalte mit ihrem Wissenserwerb zusammenhängen. Es wurden das deutschdidaktische Wissen von $N=320$ angehenden Lehrkräften mit Fach Deutsch und das erziehungswissenschaftliche Wissen von $N=1698$ angehenden Lehrkräften aller Unterrichtsfächer getestet. Die multivariaten Zusammenhänge wurden mit zwei analog spezifizierten Strukturgleichungsmodellen überprüft, die in beiden Wissensbereichen ca. $30 \%$ der Varianz aufklärten. Hypothesenkonform zeigte sich in beiden Wissensbereichen ein moderater negativer Effekt einer nichtdeutschen Familiensprache und ein kleiner positiver Effekt der Fremdsprachenkenntnisse. Eine Wechselwirkungshypothese beider Sprachvariablen wurde nur für das erziehungswissenschaftliche Wissen durch die Daten gestützt. Weiterhin ergab sich ein Zusammenhang der studentischen Nutzungsangaben mit dem Wissenserwerb von kleiner (Deutschdidaktik) oder mittlerer Effektstärke (Erziehungswissenschaft). Die Befunde werden vor dem Hintergrund eines

Dr. Armin Jentsch (Korrespondenzautor) · Prof. Dr. Jörg Doll · Dennis Meyer, Universität Hamburg, Fakultät Erziehungswissenschaft, Von-Melle-Park 8, 20146 Hamburg

E-Mail: jörg.doll@uni-hamburg.de dennis.meyer@uni-hamburg.de

Armin Jentsch arbeitet jetzt an der Universität Greifswald, Institut für Erziehungswissenschaft, Ernst-Lohmeyer-Platz 3, 17489 Greifswald, ORCID-ID: 00oo-0002-2423-3955

E-Mail: armin.jentsch@uni-greifswald.de

Ilse Stangen, Krayenkamp 5, 20459 Hamburg

E-Mail: ilse.stangen@uni-hamburg.de

Prof. Dr. Gabriele Kaiser, Universität Hamburg, Fakultät Erziehungswissenschaft, VonMelle-Park 8, 20146 Hamburg und Australian Catholic University, Institute for Learning Sciences and Teacher Education, GPO Box 2587, Brisbane Qld 4001, Australien

E-Mail: gabriele.kaiser@uni-hamburg.de 
Angebots-Nutzungs-Modells diskutiert. Sie deuten unter anderem eine große Vielfalt unterschiedlicher Sprachen bei den angehenden Lehrkräften mit nichtdeutscher Familiensprache an.

\title{
Schlagworte
}

Lehramtsstudierende, sprachbezogene Merkmale, Lerngelegenheiten, fachdidaktisches Wissen, erziehungswissenschaftliches Wissen

\section{The Importance of Language-Related Characteristics and Usage of Content Areas Learning Opportunities at University for Student Teachers' Knowledge Acquisition}

\begin{abstract}
The present study examines how language-related characteristics of student teachers and the content areas they use in learning opportunities at university relate to their knowledge acquisition. The pedagogical content knowledge of $N=320$ prospective German teachers and the general pedagogical knowledge of $N=1698$ prospective teachers of all subjects were tested. We analyzed the multivariate correlations with two analogously specified structural equation models, which explained about $30 \%$ of the variance in both knowledge domains. In accordance with our hypotheses, both knowledge domains showed a moderate negative effect of a non-German family language and a small positive effect of foreign language skills. An interaction hypothesis of both language variables was only supported by the data for general pedagogical knowledge. Furthermore, the usage of content areas in learning opportunities was related to knowledge acquisition with small (pedagogical content knowledge) or medium effect size (general pedagogical knowledge). The findings are discussed against the background of an opportunity-usage model. Amongst others, they indicate a large variety of different languages among prospective teachers with non-German family language.
\end{abstract}

\section{Keywords}

student teachers, language-related characteristics, opportunities to learn, pedagogical content knowledge, general pedagogical knowledge

\section{Einleitung}

Der Wissenserwerb während der ersten universitären Phase der Lehramtsausbildung ist eine wichtige Komponente der professionellen Entwicklung von angehenden Lehrkräften auf ihrem Weg von Noviz:innen zu Expert:innen. Er ist deshalb Gegenstand bildungswissenschaftlicher Hochschulforschung (Berliner, 2001, 2004) und wurde in Deutschland unter anderem in der Studie TEDS-LT (Teacher Education and Development Study - Learning to Teach; Blömeke, Bremerich-Vos et al., 
2013) untersucht. In TEDS-LT konnte gezeigt werden, dass das Wissen wie erwartet im Verlauf des Lehramtsstudiums zunimmt und dass ein Teil des Wissenszuwachses durch Personenmerkmale der Studierenden (z. B. Abiturnote, Migrationshintergrund, Bildungsniveau der Eltern; Blömeke, Buchholtz \& Bremerich-Vos, 2013) erklärt werden kann. Wir möchten mit dem vorliegenden Beitrag an die Ergebnisse von TEDS-LT anknüpfen, indem wir zwei Desiderate der Studie aufgreifen.

Einerseits verwendete TEDS-LT den Migrationshintergrund der Studierenden als Proxy-Variable für ihre sprachlichen Fähigkeiten und berichtete kleine negative Zusammenhänge zum Wissenserwerb in den untersuchten Fächern Deutsch, Englisch, Mathematik und Erziehungswissenschaft. ${ }^{1}$ Mit dem Migrationshintergrund können jedoch besondere Ausprägungen nicht nur in den erworbenen Sprachen, sondern u.a. auch in religiösen Einstellungen, im elterlichen Bildungsniveau und in geschlechtsbezogenen Rollenerwartungen verbunden sein.

Der Wissenserwerb im Lehramtsstudium ist bisher kaum in Abhängigkeit von differenziert gemessenen sprachbezogenen Variablen untersucht worden, obwohl etwa die Beherrschung der Wissenschaftssprache als entscheidend für erfolgreiche Lernprozesse angesehen wird (Morek \& Heller, 2012) und deshalb gerade für angehende Lehrpersonen wichtig sein dürfte. Am Beispiel der Familien- und Fremdsprachen der Studierenden untersuchen wir deshalb die Bedeutung sprachbezogener Merkmale für den Wissenserwerb von angehenden Lehrpersonen.

Des Weiteren wurde der Wissenserwerb in TEDS-LT nicht und insgesamt nur in wenigen Studien in Abhängigkeit von Merkmalen der universitären Lerngelegenheiten analysiert. Diese stellen jedoch theoretisch eine zentrale erklärende Variable für den studentischen Wissenserwerb dar (Helmke \& Schrader, 2010) und haben sich auch empirisch mehrfach als wirkmächtig gezeigt (König, Ligtvoet, Klemenz \& Rothland, 2017; Tachtsoglou \& König, 2018). Wir fokussieren in diesem Beitrag auf formale Lerngelegenheiten, also auf solche, die von Bildungsinstitutionen angeboten werden, um einen Bildungsabschluss zu erzielen (z. B. Universitäten; McDonnell, 1995; vgl. auch Tachtsoglou \& König, 2018). Wir untersuchen ihre Bedeutung für den Wissenserwerb im Lehramtsstudium und verwenden dazu die in zwei Forschungsgruppen entwickelten Itemlisten zur subjektiven Nutzung erziehungswissenschaftlicher (König, Tachtsoglou \& Seifert, 2012) und deutschdidaktischer Studieninhalte (König, Bremerich-Vos et al., 2017).

Die vorliegende Studie wurde im Rahmen eines Teilprojekts der „Qualitätsoffensive Lehrerbildung“ an einer deutschen Universität durchgeführt und analysiert den Wissenserwerb im Lehramtsstudium sowohl fachspezifisch für das fachdidaktische Wissen angehender Deutschlehrkräfte als auch fächerübergreifend für das erziehungswissenschaftliche Wissen. Beide Wissensarten sind neben dem Fachwissen wichtige Bestandteile von Modellen der professionellen Lehrerkompetenz (u. a. Baumert et al., 2010; König et al., 2018; Krauss et al., 2008).

1 In Anlehnung an Cohen (1988; vgl. auch Döring \& Bortz, 2015) sprechen wir von kleiner $(.10 \leq r<.30)$, mittlerer $(.30 \leq r<.50)$ oder großer Effektstärke $(r>.50)$. 


\section{Forschungsstand}

\subsection{Zur Bedeutung sprachbezogener Merkmale für den Wissenserwerb im Lehramtsstudium}

Zentral für die vorliegende Untersuchung ist die Rolle der Bildungssprache als „Werkzeug des Denkens“, deren Ursprung in der Zweitspracherwerbsforschung von Cummins (1984, 2008) liegt. Ein sprachbezogenes Merkmal mit Relevanz für den Erwerb der Bildungssprache ist das mehrsprachige Aufwachsen der Studierenden. Geht man davon aus, dass die Mehrheit der mehrsprachigen Studierenden Deutsch nicht als Familiensprache erlernt hat, lässt sich eine mögliche Benachteiligung im Lehramtsstudium vermuten, wie sie für die schulische Bildung empirisch belegt wurde (Klieme et al., 2010; im Überblick Lengyel, 2017).

Die wenigen nachfolgend zusammengefassten empirischen deutschsprachigen Studien zum Zusammenhang von Mehrsprachigkeit und Studienerfolg liefern hierfür erste empirische Evidenzen. In einer Studie zur Argumentationskompetenz von mehrsprachigen Studierenden und Oberstufenschüler:innen fand Petersen (2013) bei mehrsprachigen Teilnehmer:innen lexikalische Schwächen in den generierten Texten. Romero und Warneke (2012) untersuchten die akademische Schreibkompetenz Studierender. Sie fanden in diesem Merkmal zwar keine Unterschiede, allerdings begingen einsprachig Deutschsprechende nur etwa halb so viele Rechtschreib- und Grammatikfehler wie mehrsprachige Studierende. In einer Studie zur deutschsprachigen Verschriftlichung einer Bildergeschichte erhielt Jentges (2012) das Ergebnis, dass die deutschen Studierenden tendenziell weniger Fehler als die niederländischen Studierenden mit Deutsch als Zweitsprache (DaZ) machten. Das Hauptaugenmerk lenkte Jentges aber auf die satzinitiale Position des Subjekts in den Formulierungen der niederländischen Studierenden, von der diese häufigen Gebrauch machten (66\% vs. 43\%). Dieser Satzbau hatte eine geringere sprachliche Flexibilität in den Texten zur Folge.

Auch Tachtsoglou und König (2018) berichten für angehende Englischlehrkräfte einen kleinen negativen Effekt $(\beta=-.20)$ nichtdeutscher Familiensprachen auf das erziehungswissenschaftliche Wissen. Zusammenfassend betrachtet, liegen für mehrsprachige Studierende an deutschen Universitäten nur wenige Ergebnisse für meist kleine Gelegenheitsstichproben vor, die allerdings konsistent einen kleinen negativen Effekt der Mehrsprachigkeit belegen.

Ein zweites sprachbezogenes Merkmal bezieht sich auf die Fremdsprachenkenntnisse. Charakteristisch für das schulische Erlernen einer Fremdsprache ist, dass Schüler:innen mit deutscher und nichtdeutscher Familiensprache gemeinsam eine neue Sprache erlernen. Die DESI-Studie (Deutsch Englisch Schülerleistungen International; Hesse, Göbel \& Hartig, 2008) konnte zeigen, dass Schüler:innen nichtdeutscher Familiensprache beim Erlernen des Englischen überlegen waren. Für diesen Unterschied bieten sich kognitive und motivationale Erklärungen an. Mehrsprachige Schüler:innen wenden die in der außerschulischen und schulischen Sozialisation erworbenen sprachlichen Fähigkeiten und das damit verbunde- 
ne metasprachliche Wissen an, um die neue Fremdsprache zu erlernen (Lengyel, 2017). Die motivationale Erklärung betont die soziale Bedeutung der Kompetenzen in mehreren Sprachen im schulischen Kontext (Morek \& Heller, 2012). Die Schüler:innen mit nichtdeutscher Familiensprache erwerben mit dem Englischen als Fremdsprache bereits Kompetenzen in mindestens der dritten Sprache und können sich somit als sprachliche Expert:innen verstehen. Außerdem könnten sie besonders motiviert sein, eine Fremdsprache zu erlernen, da sie bereits die Nützlichkeit des Erlernens von DaZ erlebt haben. Dies ist eine Erfahrung, die sie vielen Schüler:innen mit deutscher Familiensprache voraushaben dürften.

Ganz analog können förderliche kognitive und motivationale Effekte der Fremdsprachenkenntnisse auf die Kommunikationsfähigkeit und die Ausbildung einer positiven sozialen Identität im Lehramtsstudium angenommen werden, die sich wiederum positiv auf den Wissenserwerb auswirken dürften. Während die mit der nichtdeutschen Familiensprache an einer deutschen Hochschule verbundenen sozialen Vergleichsprozesse (Frey, Dauenheimer, Parge \& Haisch, 1993) eher von dem Erleben fehlender Anerkennung durch andere Studierende begleitet sein dürften, können Fremdsprachenkenntnisse dazu beitragen, dass derartige soziale Vergleichsprozesse zur Entwicklung einer positiven sozialen Identität als Lehramtsstudierende führen.

Empirische Studien zum Zusammenhang zwischen Fremdsprachenkenntnissen und Studienerfolg liegen unseres Wissens kaum vor. In der TEDS-LT-Studie (Blömeke, Bremerich-Vos et al., 2013) zeigte sich ein kleiner positiver Effekt der Variable Zweitfach Sprache auf das deutschdidaktische Wissen. Hier scheint sich das im Zweitfach erworbene Metawissen über Sprache positiv $(\beta=.11)$ auf das deutschdidaktische Wissen auszuwirken. Auch im DaZ-Test zeigte sich bei Stangen et al. (2020) ein positiver Zusammenhang zwischen der Zahl erlernter Fremdsprachen $(r=.21)$ oder einem sprachbezogenen Studienfach $(r=.43)$ und der DaZ-Kompetenz. Da beide Studien jedoch sprachbezogenes Professionswissen untersuchten, liefern sie keine empirischen Belege für einen generellen positiven Effekt der Fremdsprachenkenntnisse auf den Wissenserwerb im Lehramtsstudium.

\subsection{Studentische Nutzungsangaben zu Lerngelegenheiten im Lehramtsstudium}

Das Forschungsparadigma der Lerngelegenheiten wurde aus dem theoretischen Ansatz der Angebots-Nutzungs-Modelle entwickelt (Helmke \& Schrader, 2010). Demnach hängt der Lernerfolg von Studierenden nicht nur von individuellen Eingangsvoraussetzungen oder universitären Rahmenbedingungen ab. Vielmehr rückt die aktive Nutzung von Lerngelegenheiten durch Studierende ins Zentrum der Forschung (Cochran-Smith \& Zeichner, 2005; Schmidt, Cogan \& Houang, 2011). Der aktiven Nutzung kommt eine vermittelnde Funktion für den Wissenserwerb zu, weil der Zusammenhang zwischen den angebotenen Lerngelegenheiten und dem 
Lernerfolg maßgeblich von den affektiv-motivationalen und kognitiven Verarbeitungsprozessen der Lernenden abhängt.

Für den Erwerb erziehungswissenschaftlichen Wissens in der Lehramtsausbildung liegen bereits Zusammenhangsanalysen mit der Nutzung universitärer Lerngelegenheiten vor. Untersucht wurden Zusammenhänge in Abhängigkeit von curricularen Unterschieden zwischen Studiengängen oder -kohorten. Kunina-Habenicht et al. (2013) verglichen das erziehungswissenschaftliche Wissen von Lehramtsstudierenden mit dem von Referendar:innen, ohne jedoch Zusammenhänge zwischen der Nutzung von Lerngelegenheiten und dem erziehungswissenschaftlichen Wissen zu finden. König, Tachtsoglou und Seifert (2012) sowie Tachtsoglou und König (2018) berichten dagegen moderate positive Zusammenhänge zwischen der Nutzung erziehungswissenschaftlicher Studieninhalte und dem erziehungswissenschaftlichen Wissen. Ähnliche Ergebnisse fanden Watson, Seifert und Schaper (2018), die außerdem die Intensität berücksichtigten, mit der die Inhalte studiert wurden. König, Ligtvoet, Klemenz und Rothland (2017) führten bei ca. 1300 Lehramtsstudierenden an 18 Universitäten in Deutschland und Österreich Zusammenhangsanalysen für die Studieninhaltsbereiche Umgang mit Heterogenität, Klassenführung/Motivierung, Strukturierung von Unterricht und Leistungsbeurteilung durch. In separaten Multilevel-Regressionsanalysen für die einzelnen Bereiche fanden sie auf der Individualebene aber lediglich signifikante Zusammenhänge zum erziehungswissenschaftlichen Wissen für den Umgang mit Heterogenität und die Strukturierung von Unterricht.

Stangen et al. (2020) konnten in einer Studie zum Zusammenhang von Lerngelegenheiten zu Mehrsprachigkeitsthemen und DaZ und der DaZ-Kompetenz von Lehramtsstudierenden zeigen, dass die Häufigkeit der Nutzung dieser Lerngelegenheiten positiv mit der Leistung im DaZ-Test (Hammer et al., 2015) korrelierte und zur Aufklärung des Kompetenzzuwachses im Verlauf eines Semesters beitrug.

Einige wenige Forschungsarbeiten haben das Paradigma der Lerngelegenheiten auf die Untersuchung der fachdidaktischen Anteile im Lehramtsstudium angewendet. Doll, Buchholtz, Kaiser, König und Bremerich-Vos (2018) verwendeten Nutzungsskalen für zentrale fachdidaktische Studieninhaltsbereiche in den Fächern Deutsch, Englisch und Mathematik und untersuchten deren curriculare Validität. Es konnte eine Zunahme der genutzten fachdidaktischen Studieninhalte im Studienverlauf gezeigt werden. König et al. (2018) untersuchten mit denselben Skalen den Zusammenhang zwischen der Nutzung fachdidaktischer Studieninhalte einerseits und dem fachdidaktischen sowie dem erziehungswissenschaftlichen Wissen von Lehramtsstudierenden in den Fächern Deutsch, Englisch und Mathematik andererseits. Trotz einer hohen Varianzaufklärung durch das Gesamtmodell konnte nur für das Fach Englisch ein mittlerer Effekt der Nutzung fachdidaktischer Studieninhalte auf das fachdidaktische Wissen gezeigt werden. Für die Fächer Deutsch und Mathematik fanden sich dagegen keine derartigen Zusammenhänge. 


\subsection{Untersuchungsmodell und Hypothesen}

Abbildung 1 präsentiert das Untersuchungsmodell, dessen Zusammenhangsannahmen im Folgenden durch fünf Hypothesen erläutert werden. ${ }^{2}$

Abbildung 1: Untersuchungsmodell zum Erwerb deutschdidaktischen Wissens.

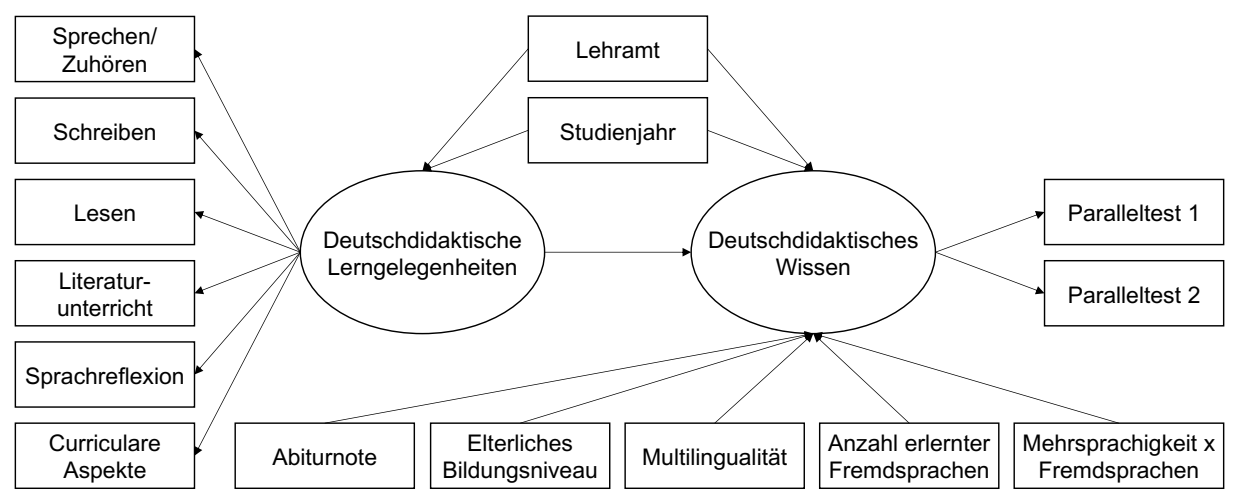

\subsubsection{Hypothesen zur Bedeutung sprachbezogener Merkmale}

Mit den Fremdsprachenkenntnissen, die durch die Proxy-Variable Anzahl erlernter Fremdsprachen operationalisiert wird, und der Familiensprache sind zwei sprachbezogene Variablen Teil des Untersuchungsmodells. Der Wissenserwerb im Lehramtsstudium setzt das Verfügen über die Bildungssprache und über wissenschaftliche Fachsprachen als kognitive Werkzeuge (Cummins, 1984, 2008; Michalak, 2014) voraus. Weiterhin kann im Hinblick auf die sozialen Beziehungen an der Hochschule die identitätsstiftende Funktion eines (fremd-)sprachlichen Expertentums die Kommunikationsfähigkeit in Lehrveranstaltungen fördern und die Integration in die Gruppe der Lehramtsstudierenden erleichtern (Frey et al., 1993; Morek \& Heller, 2012).

Hypothese 1: Wir nehmen deshalb an, dass Fremdsprachenkenntnisse den Wissenserwerb im Lehramtsstudium fördern.

Hypothese 2: Für das Vorliegen einer nichtdeutschen Familiensprache nehmen wir dagegen in Anlehnung an den skizzierten Forschungsstand einen negativen Effekt auf den deutschdidaktischen und erziehungswissenschaftlichen Wissenserwerb an.

Hypothese 3: Weiterhin nehmen wir einen Wechselwirkungseffekt zwischen der Familiensprache und den Fremdsprachenkenntnissen im Hinblick auf den Wissenserwerb an. Für Studierende mit nichtdeutscher Familiensprache vermuten wir,

2 Das analoge Modell zum Zusammenhang zwischen erziehungswissenschaftlichen Lerngelegenheiten und erziehungswissenschaftlichem Wissen wird aus Platzgründen nicht grafisch dargestellt. 
dass sich Fremdsprachenexpertise förderlicher auf den Wissenserwerb auswirkt als für solche mit Familiensprache Deutsch. Dieser Zusammenhang kann sowohl kognitiv durch umfangreiches metasprachliches Wissen als auch motivational durch die Bedeutung fremdsprachlicher Expertise für die Entwicklung einer positiven sozialen Identität als integrierte:r Lehramtsstudierende:r an einer deutschen Hochschule bei Studierenden mit nichtdeutscher Familiensprache vermittelt werden.

\subsubsection{Hypothesen zur Nutzung universitärer Lerngelegenheiten}

Zum Verständnis der Hypothesen zur Nutzung universitärer Lerngelegenheiten werden nachfolgend einige curriculare Vorgaben erläutert, die an der Universität vorliegen, an der die Studie durchgeführt wurde: (a) Der Arbeitsaufwand in der Deutschdidaktik ist zwar mit 11 ECTS-Punkten in allen Studiengängen derselbe, jedoch erwerben angehende Lehrkräfte der Primar-/Sekundarstufe I und der Sonderpädagogik im Bachelorstudium weitere 12 bzw. 6 ECTS-Punkte im fachdidaktischen Grundlagenstudium Sprache. (b) Den curricular vorgegebenen Arbeitsaufwand in der Erziehungswissenschaft verdeutlichen die folgenden ECTS-Punkte: 143 ECTS-Punkte beträgt der Studienaufwand für angehende Lehrkräfte der Sonderpädagogik, 56 für solche der Primar-/Sekundarstufe I, 48 für angehende Lehrkräfte an Berufsschulen und 40 für solche an Gymnasien (Doll et al., 2018).

Hypothese 4: Zwischen dem durch den Lehramtsstudiengang und das Studienjahr als institutionelle Merkmale curricular vorgegebenen Arbeitsaufwand in ECTS-Punkten (für curriculare Details vgl. Doll et al., 2018) einerseits und den subjektiven Nutzungsangaben zu den studierten Inhalten und dem Wissenserwerb andererseits bestehen im Sinne curricularer Validität positive Zusammenhänge.

Hypothese 5: Wir erwarten ferner, dass die von den Studierenden genutzten deutschdidaktischen bzw. erziehungswissenschaftlichen Lerngelegenheiten in dem jeweiligen Modell einen bedeutsamen Varianzanteil des Wissens aufklären, da es sich gemäß Angebots-Nutzungs-Modell (Helmke \& Schrader, 2010) um proximale Merkmale der Lernhandlungen handelt, die interindividuelle Nutzungsunterschiede berücksichtigen.

\section{Methode}

\subsection{Studiendesign und Stichprobe}

Die Daten, die für die Analysen zusammengefasst wurden, wurden in den Jahren 2016 und 2017 an einer deutschen Universität durch Online-Befragungen am Ende des jeweiligen Sommersemesters erhoben. Da das Lehramtsstudium an dieser Universität regelhaft nur im Wintersemester aufgenommen werden kann, beteiligten sich Absolvent:innen im ersten bis fünften Studienjahr. Tabelle 1 präsentiert zentrale Merkmale der beiden Untersuchungsstichproben. Es beteiligten sich $N=320$ 
Lehramtsstudierende mit dem Fach Deutsch an einem Test zum deutschdidaktischen Wissen und $N=1698$ Lehramtsstudierende aller Studienfächer an einem Test zum erziehungswissenschaftlichen Wissen. Am häufigsten vertreten sind Studierende der Lehrämter für Gymnasien bzw. für die Primar-/Sekundarstufe I. Diese beiden Studiengänge sind mit 40.8\% bzw. 41.5\% in der Stichprobe der angehenden Deutschlehrkräfte noch stärker vertreten als in der fächerübergreifenden Stichprobe (35.3\% bzw. 29.5\%).

In der fächerübergreifenden Stichprobe ist der mittlere Unterschied in den Abiturnoten zwischen ein- und mehrsprachigen Studierenden statistisch signifikant (Median $=2.1$ versus 2.4; $z=-4.45, p<.01$, Mann-Whitney- $U$-Test), für die Studierenden mit dem Unterrichtsfach Deutsch trifft dies nicht zu (Median $=1.9$ versus 2.1; $z=-1.85, p=.06$ ). Das höchste elterliche Bildungsniveau, das mit dem ISCED-97 (International Standard Classification of Education) erfasst wurde, liegt in beiden Stichproben für die einsprachig aufgewachsenen Studierenden bei dem Median $=5$ (Hochschulabschluss) und für die mehrsprachigen bei dem Median $=3$ (Abitur oder Berufsabschluss). Dieser Unterschied ist sowohl für Deutschlehramtsstudierende $(z=-2.79, p<.01)$ als auch in der fächerübergreifenden Stichprobe signifikant $(z=-5.18, p<.01)$.

Tabelle 1: Relative Häufigkeiten bzw. Mediane zu studienbezogenen und soziodemografischen Variablen der Lehramtsstudierenden (LA) in beiden Stichproben (absolute Häufigkeiten und Interquartilsabstände in Klammern)

\begin{tabular}{|c|c|c|c|c|}
\hline & \multicolumn{2}{|c|}{ Fach Deutsch $(N=320)$} & \multicolumn{2}{|c|}{ Fächerübergreifend $(N=1698)$} \\
\hline & $\begin{array}{l}\text { Einsprachig } \\
(n=272)\end{array}$ & $\begin{array}{l}\text { Mehrsprachig } \\
\quad(n=48)\end{array}$ & $\begin{array}{l}\text { Einsprachig } \\
(n=1420)\end{array}$ & $\begin{array}{l}\text { Mehrsprachig } \\
\quad(n=278)\end{array}$ \\
\hline Weiblich & $89.0 \%(242)$ & $87.8 \%(43)$ & $74.9 \%(1065)$ & $81.3 \%(226)$ \\
\hline LA Prim.-/Sek. I & $41.5 \%(113)$ & $42.8 \%(20)$ & $28.5 \%(405)$ & $36.0 \%(100)$ \\
\hline LA Gymnasien & $40.1 \%$ (109) & $42.9 \%(21)$ & $34.3 \%(488)$ & $39.9 \%$ (111) \\
\hline LA Sonderpädagogik & $8.8 \%(24)$ & $8.2 \%(4)$ & $18.3 \%(260)$ & $13.7 \%(38)$ \\
\hline LA berufliche Schulen & $9.6 \%(26)$ & $6.1 \%(3)$ & $18.9 \%(267)$ & $10.4 \%(29)$ \\
\hline Studiendauer (Jahre) & $3(2)$ & $3(2)$ & $3(2)$ & $3(3)$ \\
\hline Alter & $25(5)$ & $25(5)$ & $25(5)$ & $25(5)$ \\
\hline Abiturnote & $1.9(0.7)$ & $2.1(0.9)$ & $2.1(0.9)$ & $2.4(0.9)^{* *}$ \\
\hline ISCED-97 $7^{\mathrm{a}}$ & $5(2)^{* *}$ & $3(2)$ & $5(2)^{* * *}$ & $3(2)$ \\
\hline $\begin{array}{l}\text { Anzahl erlernter } \\
\text { Fremdsprachen }\end{array}$ & $3(1)$ & $3(1)$ & $3(1)$ & $3(1)$ \\
\hline
\end{tabular}

Anmerkungen. LA Prim.-/Sek. I = Lehramt an Primar-/Sekundarstufe I.

a Das höchste elterliche Bildungsniveau wurde mit dem ISCED-97 erfasst (o = kein Schulbesuch/Vorschule bis $6=$ Promotion/Habilitation). Kategorie $3=$ Abitur oder Berufsabschluss, Kategorie $5=$ Hochschulabschluss.

Mit dem $U$-Test wurden signifikante Unterschiede zwischen den ein- und mehrsprachigen Studierenden separat für die Deutschstudierenden und die fächerübergreifende Stichprobe getestet.

${ }^{* *} p<.01$. 
Nachfolgend werden die Stichprobenverteilungen der Deutschstudierenden und der fächerübergreifenden Stichprobe zur Untersuchung ihrer Repräsentativität in den Merkmalen Studiengang und Geschlecht mit den entsprechenden Verteilungen in der Grundgesamtheit der Lehramtsstudierenden der beteiligten Universität verglichen (in eckigen Klammern die summierten Häufigkeiten für die Grundgesamtheiten der Lehramtsstudierenden im Sommersemester 2016 und 2017). Einhundertdreiundreißig (41.5\%) [1074, 48.5\%] angehende Deutschlehrkräfte studierten das Lehramt für die Primar-/Sekundarstufe I, 130 (40.8\%) [789, 35.6\%] das Lehramt am Gymnasium, 28 (8.7\%) [154, 7.0\%] das Lehramt für Sonderpädagogik und 29 (9.0\%) [198, 8.9\%] das Lehramt an Berufsschulen. Die Anteile der Lehrämter in der Stichprobe und der Grundgesamtheit unterscheiden sich nicht signifikant, $\chi^{2}(3)$ $=6.01, p=.11$. Der Anteil von 88.7\% (284) Studentinnen mit Lehramt Deutsch verglichen mit $83.3 \%$ in der Grundgesamtheit verdeutlicht allerdings, dass Studentinnen in der Stichprobe überrepräsentiert sind, $\chi^{2}(1)=6.02, p=.01$.

Fünfhunderteins (29.5\%) [3105, 31.1\%] angehende Lehrkräfte in der fächerübergreifenden Stichprobe studierten das Lehramt für die Primar-/Sekundarstufe I, 599 (35.3\%) [3296, 33.1\%] das Lehramt am Gymnasium, 299 (17.6\%) [1657, $16.6 \%]$ das Lehramt für Sonderpädagogik und 294 (17.3\%) [1917, 19.3\%] das Lehramt an Berufsschulen. Erneut unterscheiden sich die Anteile in der Stichprobe und der Grundgesamtheit nicht signifikant voneinander, $\chi^{2}(3)=7.05, p=.07$. Verglichen mit $68.5 \%$ Studentinnen in der Grundgesamtheit ist der Anteil von $78.6 \%$ (1335) Studentinnen in der fächerübergreifenden Stichprobe allerdings ebenfalls deutlich größer, $\chi^{2}(1)=70.76, p<.01$.

\subsection{Instrumente}

\subsubsection{Sprachbezogene Merkmale}

Die sprachbezogenen Merkmale wurden mit geschlossenen und offenen Antwortformaten erfasst. Der Frage „Sind Sie mehrsprachig aufgewachsen?“ folgte ein Eingabefeld zur Angabe der entsprechenden Familiensprache(n). Bei Mehrfachnennungen wurde die erstgenannte nichtdeutsche Familiensprache berücksichtigt. Die Einteilung der Familiensprachen in Tabelle 3 des Ergebnisteils orientiert sich am online verfügbaren World Atlas of Language Structures (WALS; Dryer \& Haspelmath, 2019), der Sprachen aufgrund ihrer strukturellen Ähnlichkeit zu hierarchisch geordneten Sprachfamilien bündelt. Der anschließenden Frage „Welche weiteren Sprachen haben Sie gelernt?“ folgte die Vorgabe der vier Sprachen Englisch, Französisch, Spanisch und Latein und eines freien Feldes für die Angabe sonstiger Fremdsprachen. ${ }^{3}$ Abschließend wurden dichotome Angaben zur Sprachnutzung im

3 In der fächerübergreifenden Befragung gaben 59 von 278 mehrsprachigen Studierenden (21.2\%) ihre nichtdeutsche Familiensprache zusätzlich als erlernte Fremdsprache an. Am häufigsten wurde Englisch angegeben (16 Nennungen), gefolgt von Türkisch (14 Nennungen) und Polnisch (11 Nennungen). Die relativen Häufigkeiten fallen in der Stichprobe 
Alltag mit der Frage „Nutzen Sie in Ihrem täglichen Leben aktiv andere Sprachen als Deutsch?“ eingeleitet. Als Nutzungszwecke waren in der Familie, mit Freunden, bei der Mediennutzung und im Unterricht vorgegeben. Die Sprache(n), auf die sich die Befragten bei diesen Einschätzungen bezogen, wurden nicht erfragt.

\subsubsection{Studieninhaltsbereiche}

Den Studierenden wurden Nutzungsskalen zu zentralen Studieninhaltsbereichen vorgelegt, mit denen sie beurteilen sollten, ob ein Inhalt in ihrer Lehramtsausbildung bereits behandelt worden war oder nicht. ${ }^{4}$ In der Befragung zur Deutschdidaktik wurden sechs Studieninhaltsbereiche durch insgesamt 31 Items operationalisiert (vgl. Tabelle 2; König et al., 2017). Für diese Studieninhaltsbereiche konnte in separaten non-parametrischen IRT-Analysen Eindimensionalität und - zumindest in Ansätzen - curriculare Validität nachgewiesen werden (Doll et al., 2018). Die Reliabilitäten (vgl. Tabelle 2) können bis auf zwei Fälle als akzeptabel gelten. Im Mittel nutzten die Studierenden kohortenübergreifend $25 \%$ bis $60 \%$ der deutschdidaktischen Studieninhalte.

Die vier erziehungswissenschaftlichen Studieninhaltsbereiche Strukturierung von Unterricht, Klassenführung/Motivierung, Umgang mit Heterogenität und Leistungsbeurteilung (vgl. Tabelle 2; König et al., 2018) wurden mit insgesamt 37 Items erfasst. Wie Tabelle 2 zeigt, sind die Reliabilitäten der erziehungswissenschaftlichen Studieninhaltsbereiche gut. Für das Messinstrument liegen Nachweise kriterialer und curricularer Validität vor (z. B. Tachtsoglou \& König, 2018). Die Studierenden nutzten kohortenübergreifend im Mittel $33 \%$ bis $78 \%$ der erziehungswissenschaftlichen Studieninhalte.

der Deutschlehramtsstudierenden ähnlich aus. Wir deuten dies so, dass die Studierenden nicht nur informelle, sondern auch formale Lerngelegenheiten zum Erwerb von mündlichen und schriftlichen Fähigkeiten in ihrer nichtdeutschen Familiensprache genutzt haben. Im Sinne der obigen Ausführungen und für den Erwerb metasprachlichen Wissens (u. a. Lengyel, 2017) kann dies als wünschenswert bezeichnet werden.

4 In 2017 wurde eine Ratingskala ( 0 = gar nicht behandelt bis $6=$ umfassend behandelt) verwendet. Da sich diese und die dichotome Abfrage als weitgehend äquivalent erwiesen, wurden die Daten des zweiten Erhebungszeitpunkts für die gemeinsame Analyse dichotomisiert. 
Tabelle 2: Deutschdidaktische und erziehungswissenschaftliche Studieninhaltsbereiche mit Medianen relativer Nutzungshäufigkeiten (Interquartilsabstand in Klammern) und Reliabilitäten

\begin{tabular}{|c|c|c|c|c|}
\hline Dimension & Beispielitem & $\begin{array}{l}\text { Anzahl } \\
\text { Items }\end{array}$ & $\begin{array}{l}\text { Nutzungs- } \\
\text { häufigkeit }\end{array}$ & Cronbachs a \\
\hline \multicolumn{5}{|l|}{ Deutschdidaktik } \\
\hline Sprechen/Zuhören & Unterrichtskommunikation & 4 & $.50(.50)$ & .50 \\
\hline Schreiben & $\begin{array}{l}\text { Prozessorientierte } \\
\text { Schreibdidaktik }\end{array}$ & 8 & $.50(.50)$ & .73 \\
\hline Lesen & Förderung der Lesekompetenz & 5 & $.60(.80)$ & .81 \\
\hline Literaturunterricht & Aufgaben im Literaturunterricht & 7 & $.57(.33)$ & .76 \\
\hline Sprachreflexion & Grammatikwerkstatt & 3 & $.33(.33)$ & .53 \\
\hline Curriculare Aspekte & $\begin{array}{l}\text { Bildungsstandards im } \\
\text { Unterrichtsfach Deutsch }\end{array}$ & 4 & $.25(.75)$ & .69 \\
\hline \multicolumn{5}{|l|}{ Erziehungswissenschaft } \\
\hline $\begin{array}{l}\text { Strukturierung von } \\
\text { Unterricht }\end{array}$ & Unterrichtsmethoden & 9 & $.78(.45)$ & .78 \\
\hline $\begin{array}{l}\text { Klassenführung/ } \\
\text { Motivierung }\end{array}$ & Regeln im Unterricht & 8 & $.50(.50)$ & .76 \\
\hline $\begin{array}{l}\text { Umgang mit } \\
\text { Heterogenität }\end{array}$ & $\begin{array}{l}\text { Individuelle Förderung im } \\
\text { Unterricht }\end{array}$ & 11 & $.55(.36)$ & .76 \\
\hline Leistungsbeurteilung & Lernprozessdiagnostik & 9 & $.33(.67)$ & .83 \\
\hline
\end{tabular}

\subsubsection{Wissenstests}

Das deutschdidaktische Wissen wurde mit einem Test gemessen, der in der Studie TEDS-LT entwickelt (Bremerich-Vos \& Dämmer, 2013) und im Projekt PlanvoLL-D (Planungskompetenzen von Lehrerinnen und Lehrern im Fach Deutsch) weiterentwickelt wurde (König, Bremerich-Vos et al., 2017). Die Themengebiete Sprechen und Zuhören, Schreiben, Rechtschreiben, Lesen und Sprache sowie Sprachgebrauch reflektieren wurden mit 29 Items operationalisiert.

Zur Messung des erziehungswissenschaftlichen Wissens wurde eine Kurzfassung des in TEDS-M (Teacher Educational Development Study - Mathematics) eingesetzten Tests verwendet (König \& Blömeke, 2010). Der Test besteht aus 42 Items, die vier Wissensbereiche erfassen. Diese Wissensbereiche korrespondieren mit den erziehungswissenschaftlichen Studieninhaltsbereichen (vgl. Tabelle 2). Beide Wissenstests wurden nach dem eindimensionalen Rasch-Modell skaliert. Die Reliabilität ergab einen Wert von .87 für den Deutschdidaktik-Test und .88 für den Test zum erziehungswissenschaftlichen Wissen. Die mittlere Item-Trennschärfe betrug $r=.42$ für Deutschdidaktik und $r=.41$ für Erziehungswissenschaft. Die Studierenden lösten im Deutschdidaktik-Test im Mittel 22 Items korrekt (75.8\%), im Test des erziehungswissenschaftlichen Wissens waren es 33 Items (78.6\%). 


\subsection{Statistische Analysen}

Zur Bearbeitung der Fragestellungen wurden zwei separate Strukturgleichungsmodelle für den deutschdidaktischen und den erziehungswissenschaftlichen Wissenserwerb spezifiziert und die Modellparameter sowie die Anpassungsgüte der Modelle geschätzt (vgl. Abbildung 1). Nur das Wissen und die Nutzungsangaben zu studierten Inhalten in den Lerngelegenheiten konnten als latente Variablen operationalisiert werden, da hier mehrere Indikatoren vorlagen. Dies sind auch die beiden einzigen abhängigen Variablen. Als Indikatoren des deutschdidaktischen und erziehungswissenschaftlichen Wissens dienten je zwei Summenwerte, die über parallele Testhälften berechnet wurden. Die Indikatoren der Lerngelegenheitsnutzung bildeten Summenwerte für die Nutzungsskalen der Studieninhaltsbereiche in der Deutschdidaktik (sechs Skalen) und der Erziehungswissenschaft (vier Skalen). Die übrigen Modellvariablen, die jeweils nur durch einen Indikator operationalisiert wurden, gingen als manifeste Variablen in die Modelle ein.

Die Modellbildung erfolgte durch hierarchische Modellerweiterungen: Zunächst wurde Modell 1 geschätzt, das das Wissen der Studierenden durch institutionelle Merkmale (Studiengang und Studienjahr) und Kontrollvariablen (Abiturnote und höchstes elterliches Bildungsniveau) erklärt (u.a. Blömeke, Buchholtz et al., 2013). Dann wurde mit Modell 2 untersucht, ob die sprachbezogenen Merkmale einen zusätzlichen Beitrag zur Varianzaufklärung des Wissenserwerbs leisten können (Hypothesen 1-3). Abschließend wurden in Modell 3 die studentischen Angaben zur Nutzung der Studieninhaltsbereiche aufgenommen. Die studentischen Angaben fungieren im Modell sowohl als abhängige Variable, die durch institutionelle Merkmale aufgeklärt wird (Hypothese 4), als auch als unabhängige Variable zur Aufklärung des Wissenserwerbs (Hypothese 5).

Für alle Prädiktoren werden standardisierte Regressionskoeffizienten und Standardfehler angegeben. Zur Interpretation des Interaktionseffekts werden für beide Untersuchungsmodelle zusätzlich manifeste Korrelationen zwischen dem Wissen der Studierenden und den sprachbezogenen Merkmalen berichtet. Ein signifikanter Interaktionseffekt liegt dann vor, wenn sich die Stärke des Zusammenhangs zwischen der Anzahl erlernter Fremdsprachen und dem Wissenserwerb für ein- und mehrsprachig aufgewachsene Studierende unterscheidet.

Alle statistischen Analysen wurden mit den Softwarepaketen IBM SPSS 23 und Mplus 6 (Muthén \& Muthén, 1998-2010) durchgeführt und fehlende Werte durch die dort implementierte Full-Information-Maximum-Likelihood-Methode berücksichtigt. Zur Beurteilung der Anpassungsgüte wurden die $\chi^{2}$-Teststatistik und außerdem deskriptive Fit-Indizes bestimmt (Hu \& Bentler, 1999). Für Modellvergleiche werden zudem die Informationskriterien AIC und BIC herangezogen, bei denen ein niedrigerer Wert auf eine bessere Passung des Modells hinweist. 


\section{Ergebnisse}

\subsection{Sprachbezogene Merkmale}

Tabelle 3 zeigt, dass die gemäß WALS kategorisierten Familiensprachen der Lehramtsstudierenden ein breites Spektrum abdecken. ${ }^{5}$ Polnisch, Türkisch und Russisch und die drei Sprachfamilien iranischer, romanischer und germanischer Sprachen kommen am häufigsten vor.

Tabelle 3: Häufigkeiten in Prozent zu den sprachbezogenen Merkmalen der Studierenden mit Studienfach Deutsch und der fächerübergreifenden Stichprobe

\begin{tabular}{|c|c|c|c|c|}
\hline & \multicolumn{2}{|c|}{ Fach Deutsch $(N=320)$} & \multicolumn{2}{|c|}{ Fächerübergreifend $(N=1698)$} \\
\hline & $\begin{array}{l}\text { Einsprachig } \\
(n=271)\end{array}$ & $\begin{array}{c}\text { Mehrsprachig } \\
(n=49)\end{array}$ & $\begin{array}{c}\text { Einsprachig } \\
(n=1420)\end{array}$ & $\begin{array}{c}\text { Mehrsprachig } \\
(n=278)\end{array}$ \\
\hline \multicolumn{5}{|l|}{ Familiensprachen } \\
\hline Polnisch & - & 22.2 & - & 11.2 \\
\hline Iranische Sprachen & - & 17.8 & - & 16.7 \\
\hline Türkisch & - & 13.3 & - & 22.8 \\
\hline Russisch & - & 8.9 & - & 11.2 \\
\hline Romanische Sprachen & - & 8.9 & - & 11.2 \\
\hline Germanische Sprachen & - & 4.4 & - & 9.3 \\
\hline Andere Sprachen & - & 24.4 & - & 17.7 \\
\hline \multicolumn{5}{|l|}{$\begin{array}{l}\text { Nutzungssituationen nicht- } \\
\text { deutscher Sprachen }\end{array}$} \\
\hline ... in der Familie & 13.4 & $97.8^{* * *}$ & 14.7 & $95 \cdot 2^{* * * *}$ \\
\hline ... mit Freunden & 75.7 & 71.7 & 75.5 & $83.0^{*}$ \\
\hline ... bei der Mediennutzung & 94.2 & 87.0 & $95 \cdot 3^{* * *}$ & 83.0 \\
\hline ... im Unterricht & $47.0^{*}$ & 30.4 & $55 \cdot 3^{*}$ & 44.2 \\
\hline \multicolumn{5}{|l|}{ Erlernte Fremdsprachen } \\
\hline Englisch & 99.6 & 100 & 99.8 & 99.6 \\
\hline Französisch & $75.6^{* *}$ & 56.5 & 71.2 & 71.6 \\
\hline Spanisch & 53.8 & 50.0 & 54.1 & $65.1^{* * *}$ \\
\hline Latein & 49.5 & 43.2 & $47 \cdot 4^{* * * *}$ & 31.0 \\
\hline Andere & 28.0 & 40.6 & 30.7 & $39.6^{*}$ \\
\hline
\end{tabular}

Anmerkungen. Es wurden die Unterschiede zwischen ein- und mehrsprachigen Studierenden auf Signifikanz geprüft (jeweils separat für die Stichprobe der angehenden Deutschlehrkräfte und die fächerübergreifende).

${ }^{*} p<.05 .{ }^{* *} p<.01 .{ }^{* * * *} p<.001$.

5 Polnisch und Russisch wurden aufgrund der häufigen Nennung nicht zur slawischen Sprachenfamilie zusammengefasst. Unter den iranischen Sprachen wurden folgende Sprachen zusammengefasst: Afghanisch, Dari, Farsi, Kurdisch, Persisch und Paschto. Romanische Familiensprachen waren Italienisch, Französisch, Rumänisch und Spanisch und unter den germanischen Sprachen fanden sich Dänisch, Englisch, Friesisch, Niederländisch und Plattdeutsch. Die Kategorie sonstige Sprachen fasst Sprachen wie Arabisch, Griechisch, Twi, Vietnamesisch oder Wolof zusammen, die nur vereinzelt genannt wurden. 
Bei der aktiven Sprachnutzung fanden sich signifikante Unterschiede zwischen einund mehrsprachigen Studierenden in der Häufigkeit, mit der nichtdeutsche Sprachen (Familiensprachen und/oder Fremdsprachen) in einigen der vier vorgegebenen Situationen genutzt wurden. Die mehrsprachigen Studierenden nutzten in beiden Stichproben signifikant häufiger eine andere Sprache bzw. andere Sprachen als Deutsch sowohl im familiären Kontext (Deutsch: $\chi^{2}[1]=107.21, p<.01$; fächerübergreifend: $\left.\chi^{2}[1]=435.22, p<.01\right)$ als auch, allerdings nur fächerübergreifend, mit Freund:innen, $\chi^{2}(1)=4.90, p=.03$. Umgekehrt nutzten die einsprachigen Studierenden in beiden Stichproben eine nichtdeutsche Sprache (als Fremdsprache) häufiger im Unterricht (Deutsch: $\chi^{2}[1]=3.85, p=.05$; fächerübergreifend: $\left.\chi^{2}[1]=7.55, p<.01\right)$ und nur in der fächerübergreifenden Stichprobe auch bei der Mediennutzung, $\chi^{2}(1)=32.51, p<.01$.

Bei den Fremdsprachen wurden Unterschiede vor allem für die größere fächerübergreifende Stichprobe signifikant. So gab es bei den mehrsprachigen Studierenden einen signifikant größeren Anteil Studierender, die Spanisch, $\chi^{2}(1)=7.99$, $p<.01$, und sonstige Fremdsprachen, $\chi^{2}(1)=4.33, p=.04$, erlernt hatten. In der fächerübergreifenden Stichprobe gab es für die einsprachig aufgewachsenen Studierenden einen größeren Anteil mit Latein als erlernter Fremdsprache, $\chi^{2}(1)=$ $15.88, p<.01$, und nur in der Stichprobe der Deutschstudierenden mit Französisch als Fremdsprache, $\chi^{2}(1)=7.11, p<.01$.

\subsection{Strukturgleichungsmodelle zum Erwerb deutschdidaktischen Wissens}

Tabelle 4 präsentiert die Ergebnisse für die Strukturgleichungsmodelle zum deutschdidaktischen Wissen und Tabelle 5 zu ihrer Anpassungsgüte. Während Modell 1 die institutionellen und allgemeinen Personenmerkmale enthält, werden im Modell 2 die sprachbezogenen Personenmerkmale und im Modell 3 die Nutzungsangaben zu den deutschdidaktischen Lerngelegenheiten aufgenommen.

Modell 1 zeigt zunächst, dass mit der Studiendauer hypothesenkonform ein signifikanter Wissenszuwachs einhergeht. Ferner ergeben sich erwartungswidrige Zusammenhänge für die verschiedenen Lehramtsstudiengänge, denn nicht die angehenden Lehrkräfte der Primar-/Sekundarstufe I (Referenzgruppe in allen Strukturgleichungsmodellen) mit dem curricular vorgegebenen größten Arbeitsaufwand haben das am höchsten ausgeprägte deutschdidaktische Wissen erworben, sondern die am Gymnasium. 
Tabelle 4: Hierarchische Strukturgleichungsmodelle zum Erwerb deutschdidaktischen Wissens $(N=320)$ mit standardisierten Regressionskoeffizienten (Standardfehler in Klammern)

\begin{tabular}{|c|c|c|c|c|}
\hline & \multirow{2}{*}{$\begin{array}{c}\text { Modell } 1 \\
\text { DDWissen }\end{array}$} & \multirow{2}{*}{$\begin{array}{r}\text { Modell } 2 \\
\text { DDWissen }\end{array}$} & \multicolumn{2}{|c|}{ Modell 3} \\
\hline & & & $\begin{array}{l}\text { DDLern- } \\
\text { gelegenheiten }\end{array}$ & DDWissen \\
\hline \multicolumn{5}{|l|}{ Institutionelle Merkmale } \\
\hline LA Gymnasien $^{\mathrm{a}}$ & $.16^{*}(.07)$ & $.15^{*}(.07)$ & $-.01(.07)$ & $.15^{*}(.07)$ \\
\hline LA Sonderpädagogik ${ }^{\mathrm{a}}$ & $.04(.06)$ & $.04(.06)$ & $.04(.06)$ & $.04(.06)$ \\
\hline LA berufliche Schulen ${ }^{\mathrm{a}}$ & $-.12(.06)$ & $-.11(.06)$ & $-.11(.06)$ & $-.11(.06)$ \\
\hline Studienjahr & $.17^{* * *}(.06)$ & $.17^{*}(.06)$ & $.20^{* * *}(.06)$ & $.11(.06)$ \\
\hline \multicolumn{5}{|l|}{ Personenmerkmale } \\
\hline Abiturnote & $-.19^{* *}(.07)$ & $-.20^{* *}(.07)$ & & $-.21^{* *}(.06)$ \\
\hline Elterliches Bildungsniveau & $-.02(.06)$ & $-.05(.06)$ & & $-.06(.06)$ \\
\hline \multicolumn{5}{|l|}{ Sprachbezogene Merkmale } \\
\hline Mehrsprachigkeit ${ }^{\mathrm{b}}$ & & $-.40^{*}(.21)$ & & $-.54^{* *}(.20)$ \\
\hline Anzahl erlernter Fremdsprachen & & $.22^{* *}(.07)$ & & $.17^{* * *}(.06)$ \\
\hline $\begin{array}{l}\text { Mehrsprachigkeit } \times \\
\text { Anzahl erlernter Fremdsprachen }\end{array}$ & & $.21(.21)$ & & $.36(.21)$ \\
\hline DDLerngelegenheiten $^{\mathrm{c}}$ & & & & $.29^{* *}(.06)$ \\
\hline$R^{2}$ & .14 & .26 & .05 & .26 \\
\hline
\end{tabular}

Anmerkungen . LA = Lehramt; DDWissen = deutschdidaktisches Wissen; DDLerngelegenheiten = deutschdidaktische Lerngelegenheiten. Kontrolliert wurden außerdem das Geschlecht und das Alter. Die Nutzungskontexte anderer Sprachen als Deutsch (Tabelle 2) klärten keine zusätzliche Varianz in der abhängigen Variablen auf.

a Dummy-Kodierung, Referenzkategorie ist das Lehramt für die Primar-/Sekundarstufe I. b Einsprachigkeit $=0$, Mehrsprachigkeit $=1 .^{\mathrm{c}}$ latente Variable. ${ }^{*} p<.05 .{ }^{* *} p<.01$.

Modell 2, das die Bedeutung der sprachbezogenen Merkmale untersucht, führt annähernd zu einer Verdopplung der aufgeklärten Varianz (von 14\% zu 26\%, Tabelle 4). Es passt etwas besser zu den Daten als Modell 1, wie die Informationskriterien AIC und BIC verdeutlichen (Tabelle 5). Während sich für Fremdsprachenkenntnisse in Einklang mit Hypothese 1 ein kleiner positiver Zusammenhang $(\beta=.22$, $p<.01$ ) zeigt, ergibt sich für die Mehrsprachigkeit hypothesenkonform (Hypothese 2) ein negativer Zusammenhang von mittlerer Effektstärke ( $\beta=-.40, p=.03)$. Hypothese 3 zu einem Wechselwirkungseffekt der sprachbezogenen Merkmale wird falsifiziert $(p=.33)$.

Für Modell 3 deuten die Fit-Indizes auf eine etwas schlechtere, aber noch akzeptable Modellpassung hin (Tabelle 5). Für das Studienjahr lässt sich unter Kontrolle der Lerngelegenheiten kein direkter Zusammenhang zum Wissen mehr nachweisen. Der Effekt der Studiendauer scheint durch die Nutzung deutschdidaktischer Lerngelegenheiten vollständig vermittelt zu werden: Dazu wurde der Effekt der institutionellen Bedingungsfaktoren Studiengang und Studiendauer ge- 
mäß Abbildung 1 auch für die Nutzung der Studieninhalte bestimmt. Mit einem fortgeschrittenen Studium geht gemäß Hypothese 4 zur curricularen Validität zwar eine stärkere Nutzung der Lerngelegenheiten einher $(\beta=.20, p=.01)$, jedoch wird gleichzeitig Hypothese 4 durch fehlende Nutzungsunterschiede im Vergleich mit der Referenzgruppe der angehenden Lehrkräfte der Primar-/Sekundarstufe I falsifiziert.

Die Nutzung der Lerngelegenheiten weist in Einklang mit Hypothese 5 einen positiven Zusammenhang zum deutschdidaktischen Wissen auf $(\beta=.29, p<.01)$, der fast die Höhe einer mittleren Effektstärke von .30 erreicht. Die übrigen Zusammenhänge sind gegenüber Modell 2 weitgehend unverändert geblieben (Tabelle 4).

Tabelle 5: Anpassungsgüte dreier Strukturgleichungsmodelle zum Erwerb deutschdidaktischen Wissens

\begin{tabular}{lrrr}
\hline & Modell 1 & Modell 2 & Modell 3 \\
\hline$\chi^{2}$ & 8.57 & 12.28 & $166.99^{* * *}$ \\
$d f$ & 7 & 10 & 88 \\
CFI & .99 & .99 & .91 \\
TLI & .98 & .97 & .88 \\
RMSEA & .03 & .03 & .05 \\
SRMR & .02 & .01 & .04 \\
AIC & 3091.89 & 3056.99 & 3705.16 \\
BIC & 3144.25 & 3120.51 & 3870.97 \\
\hline
\end{tabular}

Anmerkungen . CFI = Comparative Fit Index; TLI = Tucker-Lewis Index; RMSEA = Root Mean Square Error of Approximation; SRMR = Standardized Root Mean Square Residual; AIC = Informationskriterium nach Akaike; BIC = Bayessches Informationskriterium.

${ }^{* *} p<.01$.

\subsection{Strukturgleichungsmodelle zum Erwerb erziehungswissenschaftlichen Wissens}

Tabelle 6 präsentiert die Ergebnisse für die drei Strukturgleichungsmodelle zum erziehungswissenschaftlichen Wissen und Tabelle 7 Indizes zur Anpassungsgüte der Modelle.

Während Modell 1 auch hier die institutionellen und allgemeinen Personenmerkmale enthält, werden im Modell 2 die sprachbezogenen Personenmerkmale und im Modell 3 die Nutzungsangaben zu den erziehungswissenschaftlichen Lerngelegenheiten aufgenommen.

Modell 1 klärt 23\% der Varianz des Wissens der Studierenden auf und zeigt eine hohe Anpassungsgüte. Erwartungsgemäß unterscheiden sich nur die angehenden Lehrkräfte der Sonderpädagogik mit dem höchsten curricular vorgegebenen Arbeitsaufwand signifikant von der Referenzgruppe Lehramt für die Primar-/Sekundarstufe I ( $\beta=.05, p=.05)$. Zum anderen geht mit höherer Studiendauer ein Wissenszuwachs von annähernd großer Effektstärke einher $(\beta=.46, p<.01)$. 
Tabelle 6: Strukturgleichungsmodelle zum Erwerb erziehungswissenschaftlichen Wissens $(n=1698)$ mit standardisierten Regressionskoeffizienten (Standardfehler in Klammern)

\begin{tabular}{|c|c|c|c|c|}
\hline & Modell 1 & Modell 2 & \multicolumn{2}{|c|}{ Modell 3} \\
\hline & EWWissen & EWWissen & $\begin{array}{l}\text { EWLern- } \\
\text { gelegenheiten }\end{array}$ & EWWissen \\
\hline \multicolumn{5}{|l|}{ Institutionelle Merkmale } \\
\hline LA Gymnasien $^{\mathrm{a}}$ & $-.02(.03)$ & $-.05(.03)$ & $-.17^{* *}(.03)$ & $-.04(.03)$ \\
\hline LA Sonderpädagogik ${ }^{\mathrm{a}}$ & $.05^{*}(.03)$ & $.05(.03)$ & $.09^{* *}(.03)$ & $.05(.03)$ \\
\hline LA berufliche Schulen ${ }^{\mathrm{a}}$ & $-.05(.03)$ & $-.07^{*}(.03)$ & $-.14^{* *}(.03)$ & $-.08^{* *}(.03)$ \\
\hline Studienjahr & $.46^{* *}(.02)$ & $.45^{* *}(.02)$ & $.51^{* *}(.02)$ & $.45^{* *}(.03)$ \\
\hline \multicolumn{5}{|l|}{ Personenmerkmale } \\
\hline Abiturnote & $-.05 *(.03)$ & $-.04(.03)$ & & $-.04(.02)$ \\
\hline Elterliches Bildungsniveau & $.08 * *(.02)$ & $.06 *(.03)$ & & $.05^{*}(.02)$ \\
\hline \multicolumn{5}{|l|}{ Sprachbezogene Merkmale } \\
\hline Mehrsprachigkeit ${ }^{\mathrm{b}}$ & & $-.27^{* *}(.08)$ & & $-.30^{* *}(.08)$ \\
\hline Anzahl erlernter Fremdsprachen & & $.10^{* *}(.03)$ & & $.08^{* *}(.03)$ \\
\hline $\begin{array}{l}\text { Mehrsprachigkeit } \times \\
\text { Anzahl erlernter Fremdsprachen }\end{array}$ & & $.15(.08)$ & & $.16^{*}(.08)$ \\
\hline EWLerngelegenheiten $^{\mathrm{c}}$ & & & & $.39^{* *}(.03)$ \\
\hline$R^{2}$ & .23 & .27 & .32 & .28 \\
\hline
\end{tabular}

Anmerkungen. EWLerngelegenheiten = erziehungswissenschaftliche Lerngelegenheiten, EWWissen = erziehungswissenschaftliches Wissen. Kontrolliert wurde außerdem für das Geschlecht und das Alter. Die Nutzungskontexte anderer Sprachen als Deutsch (Tabelle 2) klärten keine zusätzliche Varianz in der abhängigen Variablen auf.

a Dummy-Kodierung mit Referenzkategorie Lehramt an Primar-/Sekundarstufe I. ${ }^{\mathrm{b}}$ Einsprachigkeit $=0$, Mehrsprachigkeit $=1{ }^{\mathrm{c}}$ latente Variable.

${ }^{*} p<.05 .{ }^{* *} p<.01$.

Tabelle 7: Anpassungsgüte dreier Strukturgleichungsmodelle zum Erwerb erziehungswissenschaftlichen Wissens

\begin{tabular}{lrrr}
\hline & Modell 1 & Modell 2 & Modell 3 \\
\hline$\chi^{2}$ & 8.42 & $18.75^{*}$ & $441.62^{* *}$ \\
$d f$ & 6 & 9 & 65 \\
CFI & 1.00 & .99 & .94 \\
TLI & 1.00 & .98 & .92 \\
RMSEA & .02 & .03 & .06 \\
SRMR & .01 & .01 & .03 \\
AIC & 17480.24 & 17437.85 & 16052.42 \\
BIC & 17550.74 & 17524.79 & 16270.05 \\
\hline
\end{tabular}

Anmerkungen . CFI = Comparative Fit Index; TLI = Tucker-Lewis Index; RMSEA = Root Mean Square Error of Approximation; SRMR = Standardized Root Mean Square Residual; AIC = Informationskriterium nach Akaike; BIC = Bayessches Informationskriterium.

${ }^{*} p<.05 .{ }^{* *} p<.01$. 
Für Modell 2 zeigt sich ein Anstieg der Varianzaufklärung in der Wissensvariable um $4 \%$ auf $27 \%$ bei einer unverändert hohen Anpassungsgüte. Während sich im Modell 2 für die Fremdsprachenkenntnisse auch hier hypothesenkonform (Hypothese 1) ein kleiner positiver Zusammenhang zeigt ( $\beta=.10, p<.01$ ), ergibt sich für Mehrsprachigkeit hypothesenkonform (Hypothese 2) ein negativer Zusammenhang von kleiner Effektstärke $(\beta=-.27, p<.01)$. Es zeigt sich ein tendenzieller, aber nicht signifikanter Effekt der Wechselwirkung zwischen den beiden sprachbezogenen Variablen $(\beta=.15, p=.06)$. Die Richtung dieses Effekts ist gemäß Hypothese 3 so ausgeprägt, dass die Zahl erlernter Fremdsprachen in der Gruppe der mehrsprachigen Studierenden stärker mit dem erziehungswissenschaftlichen Wissen zusammenhängt als für die einsprachigen Studierenden (Produkt-Moment-Korrelation: $r(280)=.23$ vs. $r(1418)=.13)$.

Für Modell 3 deuten die Fit-Indizes eine schlechtere, die Informationskriterien jedoch eine deutlich bessere Anpassungsgüte an die Daten verglichen mit Modell 2 an (vgl. Tabelle 7). Hypothese 4 zur curricularen Validität wird gestützt, denn angehende Gymnasial- und Berufsschullehrkräfte geben eine signifikant geringere Nutzung ( $\beta=-.17$ bzw. $\beta=-.04$, jeweils $p<.01$ ), angehende Lehrkräfte der Sonderpädagogik eine etwas stärkere Nutzung $(\beta=.09, p<.01)$ der erziehungswissenschaftlichen Lerngelegenheiten gegenüber angehenden Lehrkräften der Primar-/ Sekundarstufe I an und mit einem fortschreitenden Studium geht eine höhere Nutzung der Lerngelegenheiten einher $(\beta=.51, p<.01)$. Die Nutzung der Lerngelegenheiten weist gemäß Hypothese 5 einen positiven Zusammenhang zum erziehungswissenschaftlichen Wissen von mittlerer Effektstärke auf $(\beta=.39, p<.01)$. Das Studienjahr zeigt weiterhin den höchsten Zusammenhang von mittlerer Effektstärke zum Wissen $(\beta=.45, p<.01)$. Die übrigen Zusammenhänge sind gegenüber dem Modell 2 weitgehend unverändert (Tabelle 6). Allerdings wird jetzt die Wechselwirkung der beiden sprachbezogenen Variablen signifikant $(\beta=.16, p=.02)$ und stützt damit Hypothese 5 .

\section{Zusammenfassung und Diskussion}

\subsection{Zur Bedeutung sprachbezogener Merkmale für den Wissenserwerb im Lehramtsstudium (Hypothesen 1-3)}

Dieser Beitrag untersuchte den deutschdidaktischen und erziehungswissenschaftlichen Wissenserwerb im Lehramtsstudium durch die in einem Angebots-Nutzungs-Modell aufgestellten Zusammenhangshypothesen (Abbildung 1). Für beide Wissensbereiche wurde hypothesenkonform (Hypothese 1) ein positiver Zusammenhang kleiner Effektstärke zu den Fremdsprachenkenntnissen der Lehramtsstudierenden gefunden. Weiterhin fanden wir hypothesenkonform (Hypothese 2) einen negativen Effekt von mittlerer bis großer Effektstärke (Deutsch: $\beta=-.53$, Erziehungswissenschaft: $\beta=-.30$ ) der Mehrsprachigkeit auf das Professionswis- 
sen der angehenden Lehrkräfte, der im Unterschied zu Schulleistungsstudien nicht durch die Berücksichtigung des elterlichen Bildungsniveaus verringert wurde, obwohl hier Unterschiede zuungunsten mehrsprachiger Studierender vorlagen (Tabelle 1). Ein möglicher Grund für die fehlende Erklärungskraft des elterlichen Bildungsniveaus dürfte in der erfolgten Selektion beim Übergang von der Schule zur Universität liegen, die zu einer Varianzeinschränkung des elterlichen Bildungsniveaus geführt haben könnte.

Zudem fanden wir zumindest für das erziehungswissenschaftliche Wissen einen kleinen Interaktionseffekt der beiden sprachbezogenen Variablen. Dieser besagt, dass mehrsprachige Studierende beim Wissenserwerb stärker von Fremdsprachenkenntnissen profitieren als einsprachige (Hypothese 3). Dieser Vorteil ist allerdings nicht so stark, dass der negative Haupteffekt der Mehrsprachigkeit kompensiert werden könnte. Für das deutschdidaktische Wissen zeigte sich zumindest tendenziell ebenfalls diese Wechselwirkung. Wegen der meist recht ähnlichen Effektgrößen in den Pfadmodellen kann angenommen werden, dass für die Stichprobe Deutschstudierender die Teststärke zur inferenzstatistischen Absicherung kleiner Effekte nicht ausgereicht hat.

Um die Bedeutung dieser sprachbezogenen Merkmale für den Wissenserwerb präziser untersuchen zu können, wäre es wünschenswert gewesen, die sprachbezogenen Merkmale genauer zu quantifizieren. Hierzu könnten beispielsweise zukünftig die erzielten Niveaus in den Fremd- und Familiensprachen in den Bereichen Verstehen und Sich-Ausdrücken in der mündlichen und schriftlichen Kommunikation erhoben werden. Hierzu wurden in der DESI-Studie entsprechende Selbsteinschätzungsskalen entwickelt (Hesse et al., 2008).

Es sei angemerkt, dass die berichteten Zusammenhänge von Mehrsprachigkeit und Professionswissen nicht so zu interpretieren sind, dass Mehrsprachigkeit Bildungserfolge generell erschwert. Eher ist es so, dass im einsprachig deutsch geprägten Bildungswesen, das der hier dokumentierten erfreulich großen Vielfalt der Familiensprachen der Lehramtsstudierenden nicht gerecht wird, mehrsprachige Lernende auch an Hochschulen gezielte Unterstützung benötigen, damit sie die positiven Auswirkungen ihrer Mehrsprachigkeit, beispielsweise beim Fremdsprachenerwerb, besser nutzen können und nicht in ihrer Bildungsbiografie benachteiligt werden. Es besteht daher ein Bedarf an Studien zur Entwicklung gezielter Unterstützungsmaßnahmen für mehrsprachige Studierende.

\subsection{Zum Zusammenhang zwischen der subjektiven Nutzung von Studieninhalten in Lerngelegenheiten und dem Wissenserwerb im Lehramtsstudium (Hypothesen 4-5)}

In beiden Modellen hing die subjektive Nutzung der Studieninhalte als unmittelbar auf die studentischen Lernhandlungen bezogenes Merkmal (Helmke \& Schrader, 2010) gemäß Hypothese 5 mit dem Erwerb des jeweiligen Professionswissens zusammen. Im Einzelnen hing das erziehungswissenschaftliche Wissen mit der 
Nutzung der korrespondierenden Studieninhaltsbereiche mit mittlerer Effektstärke zusammen ( $\beta=.39$ ), während für das deutschdidaktische Wissen nur ein kleiner Zusammenhang $(\beta=.29)$ zur Nutzung deutschdidaktischer Studieninhaltsbereiche auftrat. Zwar fiel die Messung dieser Studieninhaltsbereiche auch weniger reliabel aus (Tabelle 2) als die der erziehungswissenschaftlichen, allerdings werden derartige Unterschiede in Strukturgleichungsmodellen durch eine Reliabilitätskorrektur bei der Parameterschätzung berücksichtigt.

Erklärungen dieser unterschiedlich starken Zusammenhänge könnten (a) in Unterschieden der curricularen Validität der Messungen, (b) in Unterschieden in der inhaltlichen Korrespondenz der Items liegen, die einerseits bei der Operationalisierung der vorgegebenen Studieninhaltsbereiche und andererseits in den Wissenstests eingesetzt wurden, und (c) durch curriculare Vorgaben der untersuchten Universität begründet sein.

(a) Nur für die Nutzung der deutschdidaktischen Studieninhalte wurde die Annahme curricularer Validität teilweise falsifiziert, da sich trotz studiengangspezifisch unterschiedlicher curricularer Vorgaben keine subjektiven Nutzungsunterschiede fanden. Dies ging mit einer geringen Varianzaufklärung von $5 \%$ für die deutschdidaktischen Nutzungsangaben einher, während eine hohe Varianzaufklärung von $32 \%$ für die curricular validen erziehungswissenschaftlichen Nutzungsangaben auftrat. Dies repliziert Ergebnisse aus Doll et al. (2018) und König et al. (2018), die ebenfalls keine studiengangsbezogene curriculare Validität bzw. keinen Zusammenhang zwischen der Nutzung deutschdidaktischer Lerngelegenheiten und dem Umfang deutschdidaktischen Wissens fanden. Dieses Ergebnis deuten wir so, dass eine Überarbeitung der vorliegenden Skalen zur Messung der deutschdidaktischen Studieninhaltsbereiche ihre curriculare Validität erhöhen würde.

(b) Die Items zur Messung der Nutzung der erziehungswissenschaftlichen Studieninhalte und die zur Messung des erziehungswissenschaftlichen Wissens wurden gezielt auf die vier Bereiche Strukturierung von Unterricht, Klassenführung/ Motivierung, Umgang mit Heterogenität und Leistungsbeurteilung formuliert (König et al., 2012). Diese inhaltliche Korrespondenz ist für die sechs deutschdidaktischen Studieninhaltsbereiche und das deutschdidaktische Wissen nicht gegeben. Auch die Erhöhung der inhaltlichen Korrespondenz sollte bei einer Überarbeitung der Items zur Messung der Nutzung der deutschdidaktischen Lerninhalte angestrebt werden.

Erwartungswidrig verfügten nicht die angehenden Lehrkräfte der Primar-/ Sekundarstufe I mit der curricular höchsten Vorgabe (23 ECTS-Punkte) in der Deutschdidaktik über das größte deutschdidaktische Wissen, sondern die angehenden Lehrkräfte an Gymnasien (11 ECTS-Punkte). Möglicherweise lässt sich dieser unerwartete deutschdidaktische Wissensvorsprung durch die Annahme unterschiedlicher Prozesse bei der Formierung deutschdidaktischen Wissens in Abhängigkeit vom angestrebten Lehramt erklären, die von Krauss et al. (2008) für den Erwerb mathematikdidaktischen Wissens formuliert wurde. Sie erklärten das umfangreichere mathematikdidaktische Wissen von Gymnasiallehrkräften im Vergleich mit Lehrkräften anderer Schularten damit, dass für die Gymnasiallehrkräf- 
te die Erwerbsprozesse mathematischen Fachwissens und mathematikdidaktischen Wissens enger verbunden sind (Krauss et al., 2008; latentes $r=.96$ ) als bei den Lehrkräften anderer Schularten (latentes $r=.61$ ).

(c) Abschnitt 2.3.2 zeigt, dass an der untersuchten Universität curricular bedingt sehr unterschiedlich umfangreiche Lerngelegenheiten in der Deutschdidaktik und der Erziehungswissenschaft vorliegen. Während sich die 11 ECTS-Punkte in Deutschdidaktik auf jeweils eine Veranstaltung im Bachelor- und im Masterstudium aufteilen, wird durch das umfangreiche Angebot an erziehungswissenschaftlichen Lerngelegenheiten (mindestens 40 ECTS-Punkte) ein kumulativer Wissensaufbau in der Lehramtsausbildung stärker unterstützt. Statistisch könnten die Restriktionen der curricularen Vorgaben einerseits die Varianz der Nutzungsangaben zu deutschdidaktischen Studieninhalten vermindert und andererseits zu einem kontinuierlichen Anstieg der Nutzungsangaben erziehungswissenschaftlicher Studieninhalte im Studienverlauf geführt haben. Beide Überlegungen können erklären, warum der Zusammenhang der Nutzungsangaben zum Wissenserwerb in der Erziehungswissenschaft stärker ausgeprägt ist als in der Deutschdidaktik.

\subsection{Grenzen der Studie und Ausblick}

Die vorliegenden Ergebnisse zeigen, dass die differenzierte Erfassung studentischer Merkmale lohnenswert sein kann, wenn der Wissenszuwachs angehender Lehrpersonen untersucht wird: Im Vergleich zu einem Modell, das in Anlehnung an TEDSLT den Wissenserwerb im Wesentlichen durch institutionelle Bedingungsfaktoren beschreibt, erwiesen sich sprachliche Merkmale und subjektive Nutzungsangaben zu studierten Inhalten in formalen Lerngelegenheiten insgesamt als deutlich erklärungsmächtiger. Trotzdem konnten insgesamt nur knapp $30 \%$ der Wissensvarianz aufgeklärt werden. Daher wäre es sinnvoll, die Modelle um weitere individuelle Merkmale (z. B. kognitive Grundfähigkeiten, motivationale Merkmale, Merkmale zu Lernstrategien) zu erweitern, um ihr Erklärungspotenzial zu erhöhen.

Wir möchten zudem darauf hinweisen, dass es sich bei den Nutzungsangaben der Studierenden um Selbsteinschätzungen handelt, die durch unterschiedliche Urteilstendenzen beeinflusst sein können. Um die Validität dieser Selbsteinschätzungen und damit die Bedeutung der Befunde besser einschätzen zu können, wäre es also wünschenswert, in zukünftigen Studien auch objektivere Maße zum Studienerfolg zu berücksichtigen (z. B. besuchte Lehrveranstaltungen oder abgeschlossene Module). Ferner könnte die Erfassung der Nutzung von Studieninhalten um Analysen für informelle oder non-formale Lerngelegenheiten erweitert werden. Aus der internationalen Lehrerprofessionsforschung liegen hierzu vor allem qualitative Studien vor (z. B. Floden, 2015; Schön, 1983). Erste deutschsprachige Untersuchungen bestätigen aber, dass auch außeruniversitäre Lerngelegenheiten einen eigenständigen Beitrag zum Wissenserwerb im Lehramtsstudium leisten (Doll et al., 2020; König et al., 2018; Tachtsoglou \& König, 2018). 
Die Bedeutung von sprachbezogenen Merkmalen und Studieninhalten

\section{Literatur}

Baumert, J., Kunter, M., Blum, W., Brunner, M., Voss, T., Jordan, A., ... Tsai, Y.-M. (2010). Teachers' mathematical knowledge, cognitive activation in the classroom, and student progress. American Educational Research Journal, 47(1), 133-180. https://doi.org/10.3102/0002831209345157

Berliner, D. C. (2001). Learning about and learning from expert teachers. Educational Researcher, 35, 463-482. https://doi.org/10.1016/So883-0355(02)oooo4-6

Berliner, D. C. (2004). Describing the behavior and documenting the accomplishments of expert teachers. Bulletin of Science, Technology \& Society, 24, 200-212. https://doi.org/10.1177/0270467604265535

Blömeke, S., Bremerich-Vos, A., Kaiser, G., Nold, G., Haudeck, H., Keßler, J.-U. \& Schwippert, K. (Hrsg.). (2013). Professionelle Kompetenzen im Studienverlauf. Weitere Ergebnisse zur Deutsch-, Englisch- und Mathematiklehrerausbildung aus TEDS-LT. Waxmann.

Blömeke, S., Buchholtz, C. \& Bremerich-Vos, A. (2013). Zusammenhang institutioneller Merkmale mit dem Wissenserwerb im Lehramtsstudium. In S. Blömeke, A. Bremerich-Vos, G. Kaiser, G. Nold, H. Haudeck, J.-U. Keßler \& K. Schwippert (Hrsg.), Professionelle Kompetenzen im Studienverlauf - Weitere Ergebnisse zur Deutsch-, Englisch- und Mathematiklehrerausbildung aus TEDS-LT (S. 167-187). Waxmann.

Bremerich-Vos, A. \& Dämmer, J. (2013). Professionelles Wissen im Studienverlauf: Lehramt Deutsch. In S. Blömeke, A. Bremerich-Vos, G. Kaiser, G. Nold, H. Haudeck, J.-U. Keßler \& K. Schwippert (Hrsg.), Professionelle Kompetenzen im Studienverlauf - Weitere Ergebnisse zur Deutsch-, Englisch- und Mathematiklehrerausbildung aus TEDS-LT (S. 47-75). Waxmann.

Cochran-Smith, M. \& Zeichner, K. M. (Hrsg.). (2005). Studying teacher education: The report of the AERA Panel on Research and Teacher Education. American Educational Research Association.

Cohen, J. (1988). Statistical power analysis for the behavioral sciences. Erlbaum.

Cummins, J. (1984). Wanted: A theoretical framework for relating language proficiency to academic achievement among bilingual students. In C. Rivera (Hrsg.), Language proficiency and academic achievement (S. 2-19). Multilingual Matters.

Cummins, J. (2008). BICS and CALP: Empirical and theoretical status of the distinction. In B. V. Street \& N. H. Hornberger (Hrsg.), Encyclopedia of language and education (2. Aufl., Bd. 2: Literacy, S. 71-83). Springer.

Doll, J., Buchholtz, N., Kaiser, G., König, J. \& Bremerich-Vos, A. (2018). Nutzungsverläufe für fachdidaktische Studieninhalte der Fächer Deutsch, Englisch und Mathematik im Lehramtsstudium. Die Bedeutung der Lehrämter und der Zusammenhang mit Lehrinnovationen. Zeitschrift für Pädagogik, 64(4), 511-532.

Doll, J., Jentsch, A., Meyer, D., Kaiser, G. \& König, J. (2020). Zur Reflexion über praktische Lerngelegenheiten: Reflexionsbezogene Tätigkeiten angehender Lehrpersonen in universitären und außeruniversitären Praxisphasen. Herausforderung Lehrer_ innenbildung, 3(1), 1-17.

Döring, N. \& Bortz, J. (2015). Bestimmung von Teststärke, Effektgröße und optimalem Stichprobenumfang. In N. Döring \& J. Bortz (Hrsg.), Forschungsmethoden und Evaluation in den Sozial- und Humanwissenschaften (S. 807-866). Springer. https://doi.org/10.1007/978-3-642-41089-5_14

Dryer, M. S. \& Haspelmath, M. (2019). The World Atlas of Language Structures online. Verfügbar unter https://wals.info/languoid/family/indoeuropean\#2/25.9/38.0

Floden, R. (2015). Learning what research says about teacher preparation. In M. J. Feuer, A. I. Berman \& R. C. Atkinson (Hrsg.), Past as prologue: The National Academy of Education at 50. Members reflect (S. 279-284). National Academy of Education. 
Frey, D., Dauenheimer, D., Parge, O. \& Haisch, J. (1993). Die Theorie sozialer Vergleichsprozesse. In D. Frey \& M. Irle (Hrsg.), Theorien der Sozialpsychologie. Band I: Kognitive Theorien (S. 81-122). Huber.

Hammer, S., Carlson, S., Ehmke, T., Koch-Priewe, B., Köker, A., Ohm, U. ... Schulze, N. (2015). Kompetenz von Lehramtsstudierenden in Deutsch als Zweitsprache: Validierung des GSL-Testinstruments. Zeitschrift für Pädagogik, 61, 32-54.

Helmke, A. \& Schrader, F.-W. (2010). Hochschuldidaktik. In D. H. Rost (Hrsg.), Handwörterbuch Pädagogische Psychologie (S. 273-279). Beltz Psychologische Verlags Union.

Hesse, H.-G., Göbel, K. \& Hartig, J. (2008). Sprachliche Kompetenzen von mehrsprachigen Jugendlichen und Jugendlichen nicht-deutscher Erstsprache. In E. Klieme (Hrsg.), Unterricht und Kompetenzerwerb in Deutsch und Englisch: Ergebnisse der DESI-Studie (S. 208-230). Beltz.

Hu, L. \& Bentler, P. M. (1999). Cutoff criteria for fit indices in covariance structure analysis: Conventional criteria versus new alternatives. Structural Equation Modeling, 6, 1-55. https://doi.org/10.1080/10705519909540118

Jentges, S. (2012). Grammatisch und semantisch korrekt, aber dennoch nicht perfekt! - Schriftliche Textproduktionen niederländischer L2-Lernender des Deutschen. In U. Preußer \& N. Sennewald (Hrsg.), Literale Kompetenzentwicklung an der Hochschule (S. 234-244). Peter Lang.

Klieme, E., Artelt, C., Hartig, J., Jude, N., Köller, O., Prenzel, M. ... Stanat, P. (Hrsg.). (2010). PISA 2009. Bilanz nach einem Jahrzehnt. Waxmann.

König, J. \& Blömeke, S. (2010). Messung des pädagogischen Wissens: Theoretischer Rahmen und Teststruktur. In S. Blömeke, G. Kaiser \& R. Lehmann (Hrsg.), TEDS-M 2008: Professionelle Kompetenz und Lerngelegenheiten angehender Mathematiklehrkräfte im internationalen Vergleich (S. 253-273). Waxmann.

König, J., Bremerich-Vos, A., Buchholtz, C., Lammerding, S., Strauß, S., Fladung, I. \& Schleiffer, C. (2017). Modeling and validating the learning opportunities of preservice language teachers: On the key components of the curriculum for teacher education. European Journal of Teacher Education, 4O(2), 394-412. https://doi.org/ 10.1080/02619768.2017.1315398

König, J., Doll, J., Buchholtz, N., Förster, S., Kaspar, K., Rühl, A.-M. ... Kaiser, G. (2018). Pädagogisches Wissen versus fachdidaktisches Wissen? Struktur des professionellen Wissens bei angehenden Deutsch-, Englisch- und Mathematiklehrkräften im Studium. Zeitschrift für Erziehungswissenschaft, 21(3), 1-38. https:// doi.org/10.1007/s11618-017-0765-Z

König, J., Ligtvoet, R., Klemenz, S. \& Rothland, M. (2017). Effects of opportunities to learn in teacher preparation on future teachers' general pedagogical knowledge: Analyzing program characteristics and outcomes. Studies in Educational Evaluation, 53, 122-133. https://doi.org/10.1016/j.stueduc.2017.03.001

König, J., Tachtsoglou, S. \& Seifert, A. (2012). Individuelle Voraussetzungen, Lerngelegenheiten und der Erwerb von pädagogischem Professionswissen. In J. König \& A. Seifert (Hrsg.), Lehramtsstudierende erwerben pädagogisches Professionswissen. Ergebnisse der Längsschnittstudie LEK zur Wirksamkeit der erziehungswissenschaftlichen Lehrerausbildung (S. 234-283). Waxmann.

Krauss, S., Brunner, M., Kunter, M., Baumert, J., Blum, W., Neubrand, M. \& Jordan, A. (2008). Pedagogical content knowledge and content knowledge of secondary mathematics teachers. Journal of Educational Psychology, 10o(3), 716-725. https:// doi.org/10.1037/0022-0663.100.3.716

Kunina-Habenicht, O., Schulze-Stocker, F., Kunter, M., Baumert, J., Leutner, D., Förster, D. ... Terhart, E. (2013). Die Bedeutung der Lerngelegenheiten im Lehramtsstudium und deren individuelle Nutzung für den Aufbau des bildungswissenschaftlichen Wissens. Zeitschrift für Pädagogik, 59(1), 1-23. 
Lengyel, D. (2017). Stichwort: Mehrsprachigkeitsforschung. Zeitschrift für Erziehungswissenschaft, 2O(2), 153-174. https://doi.org/10.1007/s11618-017-0734-6

McDonnell, L. M. (1995). Opportunity to learn as a research concept and a policy instrument. Educational Evaluation and Policy Analysis, 17(3), 305-322. https:// doi.org/10.3102/01623737017003305

Michalak, M. (Hrsg.). (2014). Sprache als Lernmedium im Fachunterricht. Schneider Hohengehren.

Morek, M. \& Heller, V. (2012). Bildungssprache - Kommunikative, epistemische, soziale und interaktive Aspekte ihres Gebrauchs. Zeitschrift für Angewandte Linguistik, 57(1), 67-101. https://doi.org/10.1515/zfal-2012-0011

Muthén, L. K. \& Muthén, B. O. (1998-2010). Mplus user's guide: Statistical analysis with latent variables (6. Aufl.). Muthén \& Muthén.

Petersen, I. (2013). Entwicklung schriftlicher Argumentationskompetenz bei ein- und mehrsprachigen Oberstufenschüler/-innen und Studierenden. In H. Brandl, E. Arslan, E. Langelahn \& C. Riemer (Hrsg.), Mehrsprachig in Wissenschaft und Gesellschaft. Mehrsprachigkeit, Bildungsbeteiligung und Potenziale von Studierenden mit Migrationshintergrund (S. 69-79). Universität Bielefeld.

Romero, A. \& Warneke, F. (2012). Erfassung von Schreibkompetenzen an der Hochschule - Vergleichende Beobachtungen bei monolingualen und nicht-monolingualen Sprechern des Deutschen. In U. Preußer \& N. Sennewald (Hrsg.), Literale Kompetenzentwicklung an der Hochschule (S. 219-234). Peter Lang.

Schmidt, W. H., Cogan, L. \& Houang, R. (2011). The role of opportunity to learn in teacher preparation: An international context. Journal of Teacher Education, 62(2), 138-153. https://doi.org/10.1177/0022487110391987

Schön, D. A. (1983). The reflective practitioner - How professionals think in action. Basis Books.

Stangen, I., Schroedler, T. \& Lengyel, D. (2020). Kompetenzentwicklung für den Umgang mit Deutsch als Zweitsprache und Mehrsprachigkeit im Fachunterricht: Universitäre Lerngelegenheiten und Kompetenzmessung in der Lehrer(innen)bildung. In I. Gogolin, B. Hannover \& A. Scheunpflug (Hrsg.), Evidenzbasierung in der Lehrkräftebildung (S. 123-149). Springer VS. https://doi.org/10.1007/978-3658-22460-8_6

Tachtsoglou, S. \& König, J. (2018). Der Einfluss von Lerngelegenheiten in der Lehrerausbildung auf das pädagogische Wissen angehender Englischlehrkräfte. Journal for Educational Research Online, 10(2), 3-33.

Watson, C., Seifert, A. \& Schaper, N. (2018). Die Nutzung institutioneller Lerngelegenheiten und die Entwicklung bildungswissenschaftlichen Wissens angehender Lehrkräfte. Zeitschrift für Erziehungswissenschaft, 21(3), 565-588. https://doi. org/10.1007/s11618-017-0794-7 